\title{
On the Possibility of Chaotic Oscillations of Charge on Ferroelectric Capacitor with Negative Differential Capacity in Oscillatory Circuit with Fractal Input Voltage
}

\author{
A.A. Potapov ${ }^{1,2,4}$, I.V. Rakut ${ }^{3,4}$, A.E. Rassadin ${ }^{4}$, A.A. Tronov ${ }^{4}$ \\ ${ }^{1}$ Kotel'nikov Institute of Radio Engineering and Electronics of RAS, Moscow \\ ${ }^{2}$ JNU-IRE RAS Joint Laboratory of Information Technology and Fractal Processing of Signals, \\ JiNan University, Guangzhou \\ ${ }^{3}$ National Research Lobachevsky State University of Nizhny Novgorod, Nizhny Novgorod \\ ${ }^{4}$ Nizhny Novgorod Mathematical Society, Nizhny Novgorod, brat_ras@list.ru
}

\begin{abstract}
In the paper a new kind of integrated ferroelectric device is presented. The device is oscillatory circuit with ferroelectric capacitor with negative differential capacitance under the action of fractal input voltage. We underline that we have considered realistic model of oscillatory circuit taking into account resistance of inductance and leakage of charge from ferroelectric capacitor. Also in the paper two different architectures for generator of fractal signal have been compared, only one of them having been selected as source of input voltage. For the oscillatory circuit under investigation the Mel'nikov function has been calculated explicitly. This function proves to be alternating in sign under the wide range of its parameters. In accordance with the Mel'nikov criterion it means that under these parameters the system is the generator of chaotic electrical oscillations. In brief fractal gives rise to chaos. This theoretical conclusion has been verified in the framework of numerical simulation, estimations of orders for physical values for the sample of bilayer ferroelectric capacitor with negative differential capacitance having been done. Thus on the simplest example it has been demonstrated that synthesis of the two newest scientific directions, namely, integrated ferroelectrics and fractal radio systems, results in appearance of radio engineering devices with qualitatively new properties. The feature of the system investigated is compatibility with CMOS-technology therefore this system can be placed on a single chip. It means that it is convenient to use the described above generator of chaotic electric signals in broadband communication systems.
\end{abstract}

Keywords - homoclinic loop, split separatrix function, band-pass filter, the Weierstrass function, fractal dimension, lead zirconate-titanate, strontium titanate.

\section{INTRODUCTION}

It's well known that applying of dynamic chaos in radio electronics field is one of perspective approaches for the development of information transmission systems [1][2]. Reason of significant interest to such schemes is their wide bandwidth of chaotic signals, which allows them to provide a sufficiently high noise immunity, confidentiality and speed of information transmission [1]-[2].
The main component for building mentioned systems is chaotic signal generator, namely in [1] different schemes of such generators based on semiconductor elements has been described. However, progress in the solid state physics is gradually expanding the set of systems with the presence of dynamic chaos. Particularly in the report [3] it has been proved that chaos generator can be implemented as oscillatory circuit under harmonic voltagewith ferroelectric capacitor with negative capacity [4]-[5]. We will call such capacitor NC-capacitor.

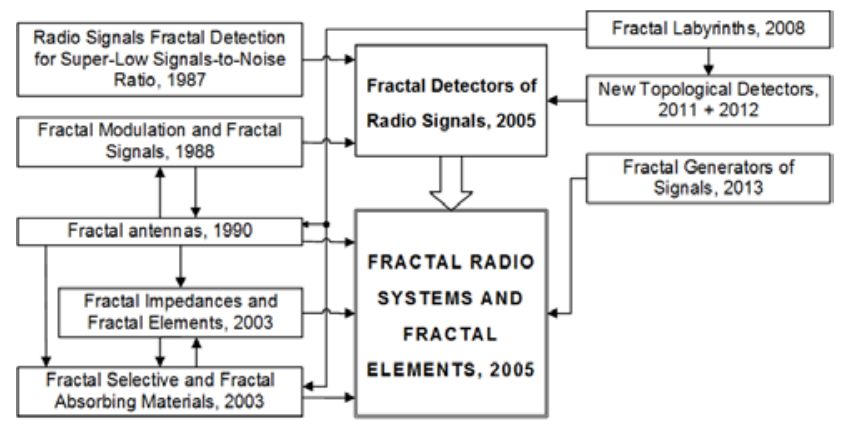

Fig. 1. The A.A. Potapov's concept "Fractal Radiophysics and Fractal Radioelectronics"

On the other hand, since the 80 s of the twentieth century in Kotel'nikov Institute of Radio Engineering and Electronics of RAS is intensively working on the creation of breakthrough information technologies in the framework of the interdisciplinary research area "Fractal Radio Physics and Fractal Radio Electronics" based on the synergistic principles of the theory of fractals, scaling effects and deterministic chaos ([6] and Fig. 1). This circumstance points to possibility for synthesis of these scientific directions, which consists in studying of fractal radio systems with NC capacitors.

Based on Melnikov criterion [7], in this article it was established that an oscillating circuit with NC capacitor under the influence of fractal signal, the circuit of which is shown in Fig. 2, demonstrates complex dynamics, and 
therefore can be used as a generator of a chaotic signal in systems of direct chaotic information transfer [1] - [2].

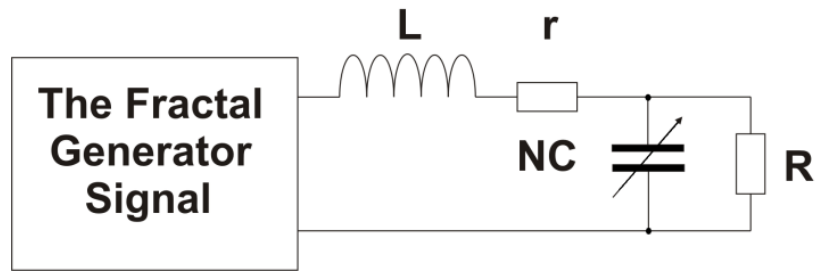

Fig. 2. Oscillating circuit with NC-capacitor under fractal voltage influence

Further, the article has next architecture: in part II we derive the equation describing oscillating circuit motion with an NC capacitor under arbitrary external voltage influence. Part III is devoted to discussing properties of fractal signals generator and calculation of separatrices splitting function (Melnikov functions). In conclusion, the obtained results and prospects for further research have been discussed.

\section{MATHEMATICAL DESCRIPTION OF OSCILLATING CIRCUIT WITH NC-CAPACITOR}

\section{A. Electrophysical properties of the NC capacitor}

The charge $q$ on both plates of NC-capacitor couples with the voltage $U_{\mathrm{NC}}$ by means of the following expression:

$$
U_{\mathrm{NC}}(q)=-\alpha \cdot q+\beta \cdot q^{3}, \alpha>0, \beta>0 .
$$

Thermodynamically stable physical realization of dependence (1) according to the results of papers [4] - [5] is possible for a heterostructure of two ferroelectrics with different Curie temperatures, but with near crystal lattices parameters. In this case, the operating temperature of all system will be located between these Curie temperatures.

From formula (1) it follows that:

$$
\frac{1}{C(q)}=\frac{\mathrm{dU}_{\mathrm{NC}}(q)}{\mathrm{dq}}=-\alpha+3 \cdot \beta \cdot q^{2},
$$

i.e. such a heterostructure has negative differential capacitance in wide range of electric charge on it. This effect was experimentally demonstrated in a two-layer system of lead zirconate-titanate $\mathrm{Pb}\left(\mathrm{Zr}_{0,2} \mathrm{Ti}_{0,8}\right) \mathrm{O}_{3}$ and strontium titanate $\mathrm{SrTiO}_{3}$ [4], and later in barium titanate system $\mathrm{BaTiO}_{3}$ the same strontium titanate $\mathrm{SrTiO}_{3}$ [5]. For the sample $\mathrm{Pb}\left(\mathrm{Zr}_{0,2} \mathrm{Ti}_{0,8}\right) \mathrm{O}_{3}$ with a thickness $28 \mathrm{~nm}$ located onSrTiO 3 with a thickness $48 \mathrm{~nm}$ and area 30 microns $\times 30$ micronsparameters $\alpha$ and $\beta$, included in the formulas (1) и (2), can be estimated as $\alpha \sim 10^{10} \mathrm{~V} / \mathrm{C}$ and $\beta \sim 0,5 \cdot 10^{29} \mathrm{~V} \cdot \mathrm{C}^{-3}[4]$.

\section{$B$. The differential equation describing the charge of NC-capacitor in oscillating circuit}

Having deal with technical applications, we will immediately consider a realistic model of oscillating circuit with NC-capacitor corresponding to the electrical circuit shown in Fig. 2, namely, we will take into account not only the small resistance inductance $r$, but also a charge leak $q$ of NC-capacitor by means of large resistance $\mathrm{R}$ connected in parallel.

The first Kirchhoff's law for scheme on Fig. 2 gives:

$$
\frac{\mathrm{dq}}{\mathrm{dt}}=I_{r}+I_{R}
$$

where $I_{r}$ and $I_{R}$ are currents through resistance $r$ and $R$ accordingly.

The second Kirchhoff's law for closed loop L-r-NC shown in Fig. 2 is given by means of:

$$
L \cdot \frac{\mathrm{dI}_{r}}{\mathrm{dt}}+r \cdot I_{r}+U_{\mathrm{NC}}(q)=U(t),
$$

where $U_{\mathrm{NC}}(q)$ is given by (1), voltage $U(t)$, provided by generator will be described in the next part.

The second Kirchhoff's law for closed loop R-NC shown in Fig. 2 is given by means of:

$$
R \cdot I_{R}+U_{\mathrm{NC}}(q)=0 .
$$

Combining equations (3)-(5), we can find that charge of NC-capacitor satisfies the nonlinear nonstationary ordinary differential equation of the 2nd order:

$$
L \cdot \frac{d^{2} q}{\mathrm{dt}^{2}}+\left(1+\frac{r}{R}\right) \cdot U_{\mathrm{NC}}(q)+\left[r+\frac{L}{R \cdot C(q)}\right] \cdot \frac{\mathrm{dq}}{\mathrm{dt}}=U(t) .
$$

Let's assume that $\alpha \cdot L / R<r, r<<R$, and introduce the following dimensionless value:

$$
\begin{gathered}
\tau=\sqrt{\frac{\alpha}{L} \cdot\left(1+\frac{r}{R}\right)} \cdot t, x=\sqrt{\frac{\beta}{\alpha}} \cdot q, \\
\delta=\frac{1}{2 \cdot \sqrt{\alpha \cdot L}} \cdot \frac{1}{\sqrt{1+r / R}} \cdot\left(r-\frac{\alpha \cdot L}{R}\right), \\
\gamma=\frac{1}{R} \cdot \sqrt{\frac{\alpha \cdot L}{1+r / R}}, u(\tau)=\sqrt{\frac{\beta}{\alpha^{3}}} \cdot \frac{U(t)}{1+r / R} .
\end{gathered}
$$

The physical meaning of the dimensionless variables and parameters (7) is that with their help we see the scales of physical quantities in the problem under study, for example, the characteristic scale of the charge on the NC capacitor from zirconate-lead titanate and strontium titanate is $\sqrt{\alpha / \beta} \sim 0,5 \mathrm{nC}$.

Let's substitute the variables (7) into equation (6), in this case we get:

$\ddot{x}-x+x^{3}=-2 \cdot \delta \cdot \dot{x}-3 \cdot \gamma \cdot x^{2} \cdot \dot{x}+u(\tau)$,

where the dot means differentiation by dimensionless time $\tau$.

Dimensionless variables form (8) is more convenient than dimension variables form (6). Moreover, view (8) makes it possible to easily distinguish effects caused by the imperfection of system's elements and external influence, namely they are all concentrated in the right side of the equation.

Consideration of real system Fig. 2 can be got by means of next transitions $r \rightarrow 0$ and $R \rightarrow \infty$ in formulas (7). It means that $\delta \rightarrow 0$ and $\gamma \rightarrow 0$. 


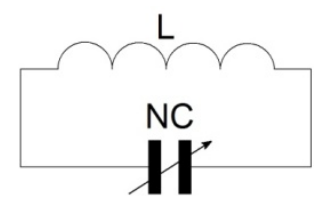

Fig. 3. Ideal RLC circuit with ideal NC-capacitor

Further, disabling the external voltage source we obtain that equation (8) can be reduced to the system of two ordinary differential equations:

$$
\dot{x}=y, \quad \dot{y}=x-x^{3} .
$$
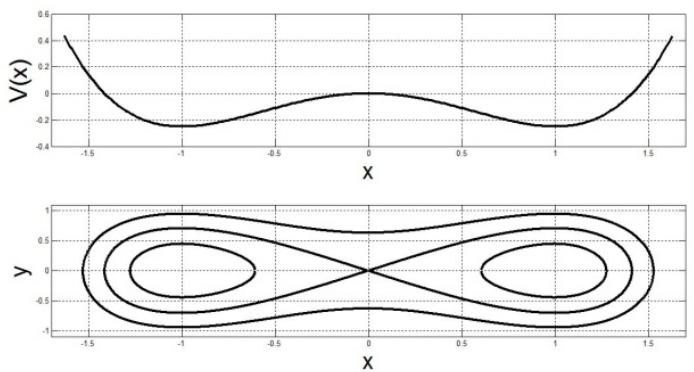

Fig. 4. The phase diagram of ideal oscillatory circuit based on ideal NC-capacitor

System (9) is a Hamiltonian one with Hamiltonian [8]:

$$
H(x, y)=\frac{y^{2}}{2}+V(x),
$$

corresponding to one-dimensional motion in potential $V(x)=-x^{2} / 2+x^{4} / 4$ of material point with elementary mass.

Phase diagrams of system (9) are shown at Fig. 4 in the bottom. They are level lines of the hamiltonian (10).

For further, it has fundamental meaning that the zero level of this Hamiltonian, which defines homoclinic loops.

These separatrices are parametrized as follows [8]:

$$
X(\tau)= \pm \frac{\sqrt{2}}{\operatorname{ch}\left(\tau+\tau_{0}\right)}, \quad Y(\tau)=\mp \frac{\sqrt{2} \cdot \operatorname{sh}\left(\tau+\tau_{0}\right)}{\operatorname{ch}^{2}\left(\tau+\tau_{0}\right)},
$$

where $\tau_{0}$ - arbitrary time shift along the homoclinic loop.

\section{FUNCTION OF SPLITTING SEPARATRICES FOR AN} OSCILLATING CIRCUIT WITH AN NC - CAPACITOR UNDER THE INFLUENCE OF FRACTAL VOLTAGE

By means of the hamiltonian (10) the equation (8) can be modified as a quasi-Hamiltonian system:

$$
\dot{x}=\frac{\partial H(x, y)}{\partial y} \quad \dot{y}=-\frac{\partial H(x, y)}{\partial x}+f(x, y, \tau),
$$

where

$$
f(x, y, \tau)=-2 \cdot \delta \cdot y-3 \cdot \gamma \cdot x^{2} \cdot y+u(\tau) .
$$

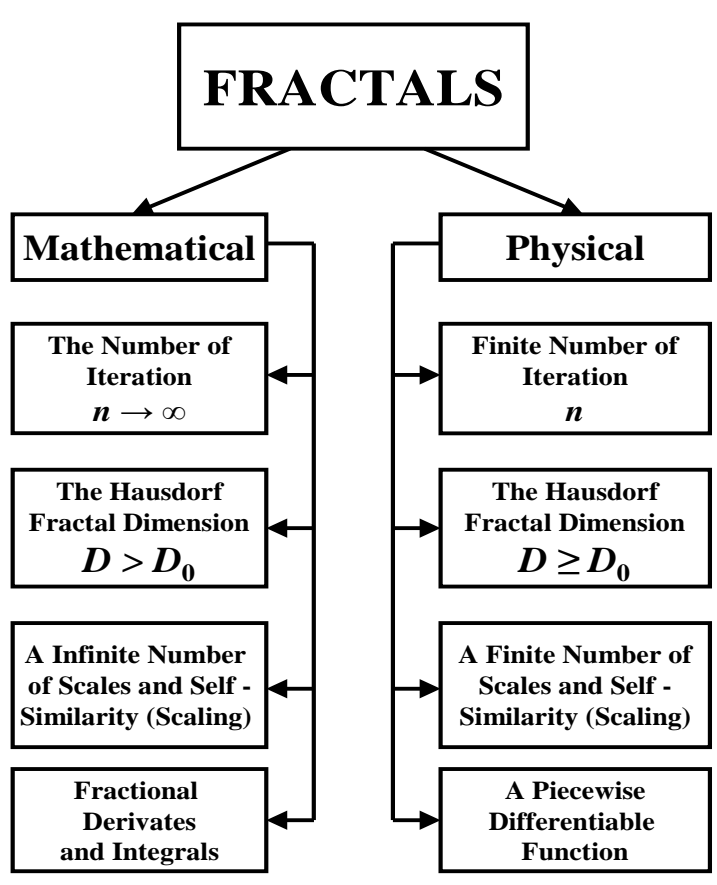

Fig. 5. Fractal sets classification according to A.A. Potapov

For clarifying complicated behavior existing in system (12), by means of Melnikov's criteria [7], function (13) has to be periodic in dimensionless time $\tau$ with a period $\theta: f(x, y, \tau+\theta)=f(x, y, \tau)$. It means, that output signal (Fig. 2) has to be periodic in physical time $t$ with period $T$ : $U(t+T)=U(t)$, and the dimensionless period $\theta$ is associated with the dimensional period $T$ by means of the first formula (7).

To specify output voltage form $U(t)$, let's have a look at the Weierstrass function:

$$
W(t)=\sum_{n=0}^{\infty} a^{n} \cdot \cos \left(b^{n} \cdot \omega_{0} \cdot t\right),
$$

where $\omega_{0}-$ value with the dimension of the cyclic frequency, with the values of the parameters $b>1,0<$ $a<1$ and $a \cdot b \geq 1$ with fractal dimension $D=2+$ $\ln a / \ln b$ having a derivative nowhere [6].

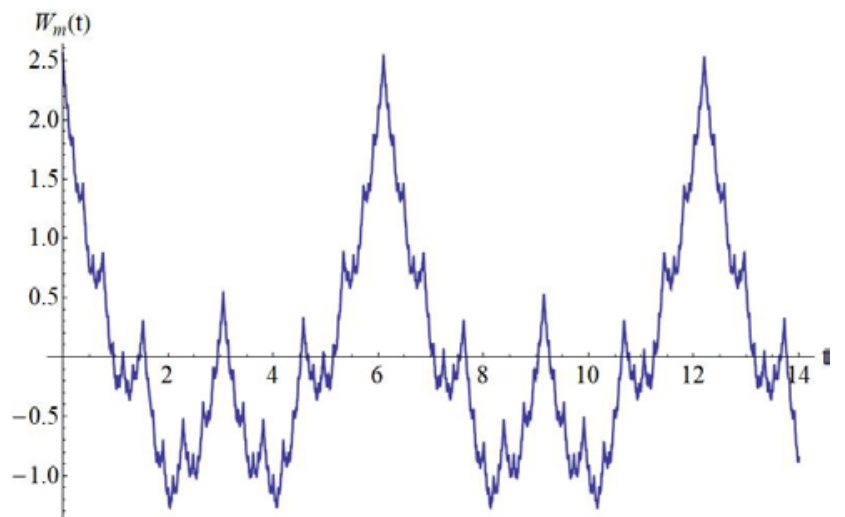

Fig. 6. Weierstrass Physical Function 
In order for the function (14) to be periodic with $T=$ $2 \cdot \pi / \omega_{0}$, value of $\mathrm{b}$ has to be $b=2,3,4, \ldots$

Since it is so difficult to implement summation of the infinite series (14) in the microelectronic system, following the Potapov's principle [6], we are going to take into account only the first $m$ terms of the sum :

$$
W_{m}(t)=\sum_{n=0}^{m} a^{n} \cdot \cos \left(b^{n} \cdot \omega_{0} \cdot t\right) .
$$

We call this sum the Weierstrass physical function (WPF) . It's easy to see that for a given accuracy $\delta>0$ and with the number of members of the sum (15) $m>$ $\ln [\delta \cdot(1 / a-1)] / \ln a$ at any time moment $t$ :

$$
\left|W(t)-W_{m}(t)\right|<\delta .
$$

In Fig. 6 you can see WPF behavior at $a=0,71, b=$ 2 and $\omega_{0}=1.03 \mathrm{~s}^{-1}$. It's periodic with $T \approx 6.1 \mathrm{~s}$ and has fractal dimension $D \approx 1.51$. In (15) we have chosen $m=$ 17, that corresponds to $\delta=10^{-2}$. From (16) it follows, that with these parameter values the Weierstrass mathematical function (14) is located in a strip of thickness $2 \cdot \delta$.

Next, we will consider variants of the fractal signals generators with output voltage for given values $a, b$, $\omega_{0}$ and $\delta$ :

$$
U(t)=U_{0} \cdot W_{m}(t),
$$

where $U_{0}$ - characteristic scale of the generator output voltage .

\section{A. Selection of the optimal Weierstrass physical function generator}

One of possible scheme implementations (17), suggested in report [9], is shown in Fig. 7. Its feature is the generation of each harmonic with frequency $\omega_{0} \cdot b^{n}$, included in (17), with separate Van Der Pol generator (Fig. 8). Nonlinear distortion of the signal due to the small difference in the limit cycle of the generator can be removed by bandpass filter. Summary voltage (17) can be obtained by means of an operational amplifier as it's shown Fig. 7.

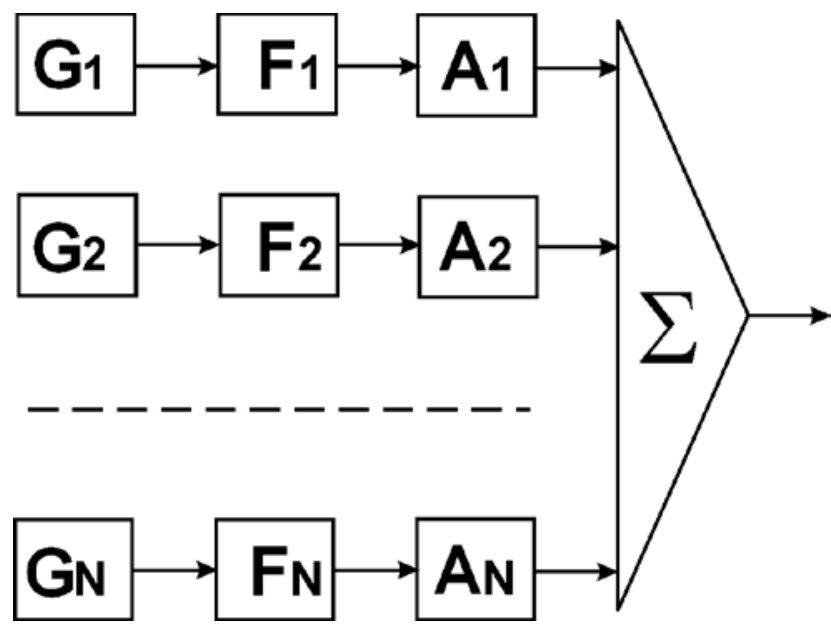

Fig. 7. Block diagram of the Weierstrass physical function generator based on Van der Paul generators

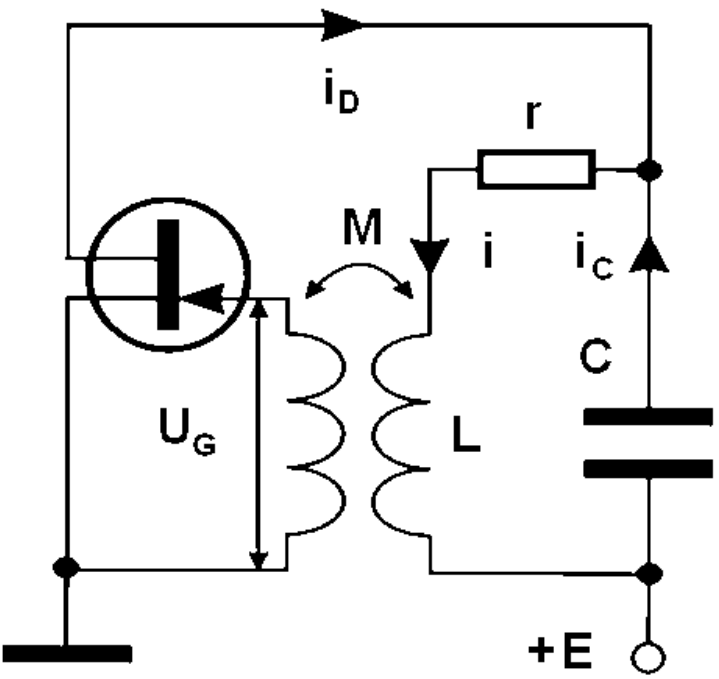

Fig. 8. The electric circuit of the Van der Pol generator on field-effect transistor

It's clear from Fig. 8 that cyclic frequency can be implemented as geometric frequency progression. However, in practice, the frequencies $\omega_{n}$ may be very different from the values $\omega_{0} \cdot b^{n}$ due to the spread of inductance values and capacitances, which leads to the effect of quasi-periodicity of the output voltage, significantly complicating the theory ([10] and references there). Besides, each generator gives a random phase shift due to voltage fluctuations in the field-effect transistor [9]. Thus, to exclude these phases from the output voltage of the generator in Fig. 7 additional measures are required.

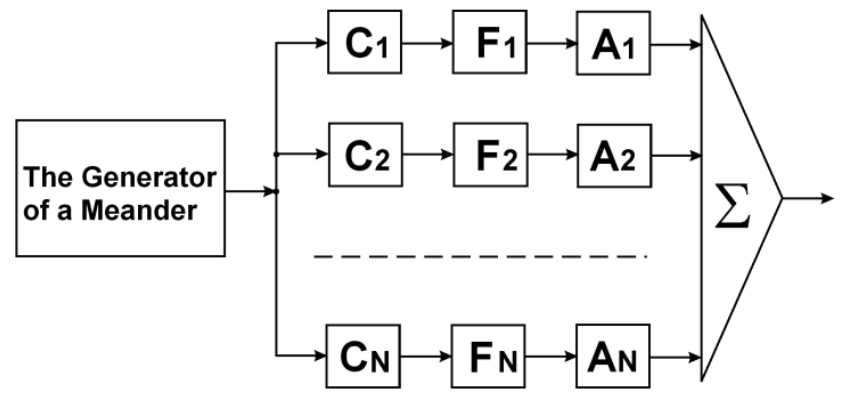

Fig. 9. Block diagram of the Weierstrass physical function generator based on the meander generator

Free from the above disadvantages is the generator circuit (Fig. 9), described in the patent [11].

Input of each of the frequency dividers (Fig. 9) receives periodic signal with period $T_{0}$ :

$$
V_{0}\left(t, T_{0}\right)=\left\{\begin{array}{cc}
U_{0}, & 0 \leq t<T_{0} / 2 \\
0, & T_{0} / 2 \leq t<T_{0}
\end{array} .\right.
$$

This action causes output voltage on frequency dividers(implemented based on JK-triggers):

$$
V_{k}(t)=V_{0}\left(t, T_{k}\right), \quad k=\overline{1, N},
$$

which repeats signal form (18), but with increasing periods in accordance with $T_{k}=T_{0} \cdot 2^{k}$. 
Each input signal (19) has the following Fourier series expansion:

$$
V_{k}(t)=\frac{U_{0}}{2}+\sum_{l=1}^{\infty} \frac{2 \cdot U_{0}}{\pi \cdot(2 \cdot l-1)} \cdot \sin \frac{2 \cdot \pi \cdot(2 \cdot l-1) \cdot t}{T_{k}} .
$$

Further, applying voltage (19) to a bandpass filter tuned to the frequency $2 \cdot \pi / T_{k}$ and provided phase shift $\pi / 2$, we can select only the first harmonic, and the frequencies of these harmonics form a geometric progression with denominator $b=2$.

Finally, adjusting the amplitudes of these harmonics properly with the help of amplifiers with adjustable gain, we get required signal (17) at the output of the adder in Fig. 9 .

\section{B. The separatrix splitting function finding}

According to common approach developed in [7], for the quasi-Hamiltonian system (12), the splitting function of the separatrices (Melnikov's function) are:

$$
\Delta\left(\tau_{0}\right)=\int_{-\infty}^{+\infty} \Upsilon(\tau) \cdot f(X(\tau), \Upsilon(\tau), \tau) \cdot d \tau,
$$

where $f(x, y, \tau)$ is function (13), its arguments are explicit expressions (11) for separatrices of the system (9).

Function $u(\tau)$ existing in (13) can be written as: where

$$
u(\tau)=A_{0} \cdot \sum_{n=0}^{m} a^{n} \cdot \cos \left(2^{n} \cdot \Omega_{0} \cdot \tau\right),
$$

$$
A_{0}=\sqrt{\frac{\beta}{\alpha^{3}}} \cdot \frac{U_{0}}{1+r / R}, \Omega_{0}=\frac{\omega_{0}}{\sqrt{1+r / R}} \cdot \sqrt{\frac{L}{\alpha}} .
$$

Thus from expressions (21) and (22) we can obtain the following expression for the separatrix splitting function of the system (12) :

$$
\begin{gathered}
\Delta\left(\tau_{0}\right)=-\frac{8 \cdot \delta}{3}-\frac{16 \cdot \gamma}{5}- \\
-\sum_{n=0}^{m} \frac{\sqrt{2} \cdot \pi \cdot A_{0} \cdot \Omega_{0} \cdot(2 \cdot a)^{n}}{\operatorname{ch}\left(\pi \cdot \Omega_{0} \cdot 2^{n-1}\right)} \cdot \sin \left(2^{n} \cdot \Omega_{0} \cdot \tau_{0}\right)
\end{gathered}
$$

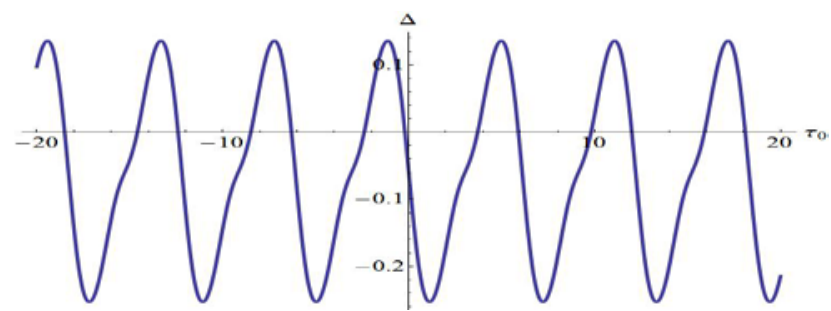

Fig. 10. Melnikov's function

Melnikov's function (24) shows how far the separatrixes of the unperturbed system (9) diverges under the action of a perturbation (13). Fig. 10 gives function graphic (24) at the following parameters: $a=0.71, b=$ $2, m=17, \Omega_{0}=1.03, A_{0}=0.1, \delta=\gamma=0.01$. As it can be seen from this graphic, at these parameters, the Melnikov function is alternating, which means that, in accordance with the Melnikov criterion [7], the system has to have chaotic behavior.
In Fig. 11 there is a graph of dimensionless NCcapacitor voltage (1), depending from dimensionless time $\tau$ at the same parameters. Graphic on Fig. 11 has been obtained by numerical solution of system (12) with initial conditions $x(0)=1.43$ and $y(0)=0.1$. From this graph it follows that the dynamics of voltage across the NCcapacitor has difficult behavior in full compliance with Melnikov's criterion [7].

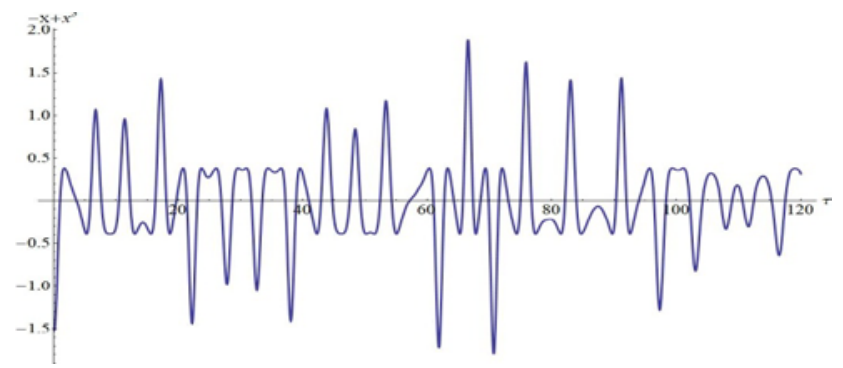

Fig. 11. The dimensionless voltage on the NC-capacitor

If the NC capacitor is made of lead zirconate titanate and strontium titanate [4], then the characteristic voltage scale on it is $\sqrt{\alpha^{3} / \beta} \sim 5 \mathrm{~V}$. It means that according to the graph in Fig. 11 peak values of the dimensional voltage on the NC-capacitor $U_{\mathrm{NC}} \sim 50 \quad \mathrm{~V}$. For selected parameters $\delta$ and $\gamma$ according to (7) $r / R \sim 10^{-4}$, therefore, due to the first of formulas (23), the characteristic amplitude of output voltage produced by generator (Fig. 9) is $U_{0} \sim 5 \mathrm{~V}$, that is quite appropriate for digital electronics.

\section{CONCLUSION}

In the article we present the explicit form of the separatrix splitting function (24) for an oscillatory circuit with an NC-capacitor, which is under the influence of a fractal input voltage. In accordance with computational experiment this function remains alternating in a wide parameter values range, therefore, the quasi-Hamiltonian system (12) will exhibit chaotic behavior with such parameters. Briefly, the essence of the observed effect can be described as follows: the fractal creates chaos. Therefore, the system in Fig. 2. can be considered as a chaotic electrical signal generator.

All over the world, interest in NC-capacitors is caused by the fact that their use as a gate dielectric in field-effect transistors makes it possible to reduce the energy consumption of microprocessors on such transistors by an order of magnitude as compared to microprocessors on conventional transistors [12]. However, the fact is overlooked that a NC-capacitor makes it possible to create a new generation of radio engineering devices with nontrivial features in their operation, which both this article and [3] - [8] demonstrate.

\section{SUPPORT}

This work was supported by the RFBR grant №18-08-01356-a. 


\section{REFERENCES}

[1] Dmitriev A.S., Efremova E.V., Maksimov N.A., Panas A.I. Generatsiya haosa (Generation of chaos) / Pod red. A.S. Dmitrieva. M.: Tehnosfera. 2012. 436 s. (in Russian)

[2] Leonov K.N., Potapov A.A., Ushakov P.A. Application of invariant properties of chaotic signals in the synthesis of noise-immune broadband systems for data transmission // Journal of Communications Technology and Electronics. 2014. V. 59. N. 12. P. 1393-1411.

[3] PotapovA.A., RassadinA.E., Tronov A.A.Generators of Chaotic Electrical Oscillations on Basis of Ferroelectric Capacitor with a Negative Capacitance // Proc. $14^{\text {th }}$ Sino Russia Symposium on Advanced Materials and Technologies / Ed. Mingxing Jia (Sanya, Hainan Province, China: November 28 - December 1, 2017).- Beijing: Metallurgical Industry Press (China, http://www.cnmip.com.cn). 2017. P. 487 - 490. (ISBN 9787-900853-01-1). (access date: 22.03.2018)

[4] Khan A.I., Bhowmik D., Yu P., Kim S.J., Pan X., Ramesh R., Salahuddin S. Experimental evidence of ferroelectric negative capacitance in nanoscale heterostructures // Applied Physics Letters. 2011. V. 99. N. 11. P. 113501-1-3.

[5] Appleby D.J.R., Ponon N.K., Kwa K.S.K., Zou B., Petrov P.K., Wang T., Alford N.M., O’Neill A.Experimental Observation of Negative Capacitance in Ferroelectrics at Room Temperature // Nano Letters. 2014. V. 14. N. 7. P. 3864-3868.

[6] Potapov A.A. Fractali $v$ radiofizike i radiolokatsii: Topologija viborki (Fractals in radiophysics and radiolocation: Sample Topology). M.: Universitetskaya kniga. 2005. 848 s. (in Russian)

[7] Melnikov V.K. Ob ustoichivosti centra pri periodicheskih po vremeni vozmusheniyah (On stability of center under periodic on time perturbations) // Trudi Moskovskogo matematicheskogo obshestva. 1963. T. 12. S. 3 - 52. (in Russian)

[8] Kostromina O.S., Potapov A.A., Rakut I.V., Rassadin A.E. Total harmonic distorsions in an oscillatory circuit with a ferroelectric capacitor with a negative capacitance // Eurasian Physical Technical Journal. 2017. V. 14. N 2 (28). P. 14-21.

[9] Potapov A.A., Rakut I.V., Rassadin A.E., Stepanov A.V., Tronov A.A. Generator of fractal voltage: practical scheme // International conference on Dynamical Systems 'Shilnikov WorkShop 2017' (Nizhny Novgorod, Russia, December 15-16, 2017). http://www.shilnikov.unn.ru/en(access date: 22.03.2018). Book of abstracts. P. 28.

[10] Morozov A.D., Morozov K.E. O kvaziperiodicheskih vozmusheniyah dvumernih gamil'tonovih sistem (On quasiperiodic perturbations of two-dimensional Hamiltonian systems) // Differentsial'nie uravneniya. 2017. T. 53. № 12. S. 1607-1615.

[11] Kudasov Yu.B., Makarova N.N., Dubinov A.E. Generator fraktalnogo signala (Generator of fractal signal). 2001. Patent RF 2168848.

[12] Rollo T., Esseni D. New design perspective for Ferroelectric NC-FETs // IEEE Electron Device Letters. January 2018. P. 1-1. 